\title{
An improved authenticated key agreement protocol for telecare medicine information system
}

\author{
Wenhao Liu, Qi Xie ${ }^{*}$, Shengbao Wang and Bin Hu
}

${ }^{*}$ Correspondence: qixie68@126.com

Hangzhou Key Laboratory

of Cryptography

and Network Security,

Hangzhou Normal University,

Hangzhou 311121, China

\begin{abstract}
In telecare medicine information systems (TMIS), identity authentication of patients plays an important role and has been widely studied in the research field. Generally, it is realized by an authenticated key agreement protocol, and many such protocols were proposed in the literature. Recently, Zhang et al. pointed out that Islam et al.'s protocol suffers from the following security weaknesses: (1) Any legal but malicious patient can reveal other user's identity; (2) An attacker can launch off-line password guessing attack and the impersonation attack if the patient's identity is compromised. Zhang et al. also proposed an improved authenticated key agreement scheme with privacy protection for TMIS. However, in this paper, we point out that Zhang et al's scheme cannot resist off-line password guessing attack, and it fails to provide the revocation of lost/stolen smartcard. In order to overcome these weaknesses, we propose an improved protocol, the security and authentication of which can be proven using applied pi calculus based formal verification tool ProVerif.
\end{abstract}

Keywords: Authentication, Protocol, Biometrics, Smart card

\section{Background}

In Internet environment, especially in the $\mathrm{C} / \mathrm{S}$ model, it is crucial to authenticate both the user and the server when the user needs to access services provided by the server (Khan et al. 2014). The telecare medicine information system (TMIS) has attracted great attention of researchers to establish a convenient communication over the Internet between patients at home and doctors at a clinical center or home health-care agency (Kaul and Awasthi 2013; Wen 2013). A doctor can easily get access to his patient's medical history from TMIS, and diagnose quickly without repeating physical examination. Besides, TMIS can save the patients' expenses and time (Xie et al. 2014). However, it is a great challenge to preserve the security and privacy of patient's information transmitted over the Internet (Xie et al. 2013; Siddiqui et al. 2014).

\section{Related works}

Wu et al. (2010) proposed the first two-factor authentication scheme for TMIS service. Since then, a lot of two-factor authentication protocols have been proposed (He et al. 2012; Wei et al. 2012; Zhu 2012; Muhaya 2015). He et al. (2012) showed that Wu et al's

(c) 2016 Liu et al. This article is distributed under the terms of the Creative Commons Attribution 4.0 International License (http:// creativecommons.org/licenses/by/4.0/1, which permits unrestricted use, distribution, and reproduction in any medium, provided you give appropriate credit to the original author(s) and the source, provide a link to the Creative Commons license, and indicate if changes were made. 
protocol could not resist insider attack and impersonation attack. And they gave an improved protocol using smartcard. However, Wei et al. (2012) showed that He et al's protocol failed to resist off-line password guessing attack, and they also proposed an improved scheme, but Wei et al's scheme has the same security defects. In order to fix the above drawbacks, Zhu (2012) proposed an improved scheme. Unfortunately, Zhu et al's scheme has been proven insecure by Muhaya (2015). Wu et al. (2012) proposed a password-based user authentication scheme for the integrated EPR information system. Later, Islam and Biswas (2014) found that Wu et al's (2012) scheme cannot resist privileged-insider attack, off-line password guessing attack and ephemeral secret leakage attack.

It's an interesting topic to improve security and computation efficiency of the authentication schemes. Pu et al. (2010) designed an anonymous authentication scheme for TMIS service using the elliptic curve cryptography (ECC). Chen et al. (2012) proposed a dynamic-identity based authentication scheme for TMIS. However, Jiang et al. (2013) showed Chen et al's scheme (Chen et al. 2012) cannot withstand impersonation attack, off-line password guessing attack and denial-of-service attack. Recently, Xu et al. (2014) proposed a two-factor authentication key agreement protocol using ECC. Unfortunately, Islam and Khan (2014) showed that Xu et al.s scheme (Xu et al. 2014) can neither withstand replay attack, nor provide the revocation of lost/lost smart or achieve strong authentication in login and authentication phases. In order to overcome the above defects, they proposed a new anonymous two-factor authentication protocol for TMIS. Recently, Zhang and Zhou (2015) pointed out that Islam et al's protocol has many security defects such as: (1) Any legal but malicious patient can reveal other user's identity; (2) An attacker can launch off-line password guessing attack and the impersonation attack if he knows legal user's identity. Zhang et al. then proposed a new ECC-based authenticated key agreement scheme in order to fix the above security problems. In 2015, Chaudhry et al. (2015) also showed that Islam et al's protocol (Islam and Khan 2014) suffers from user impersonation attacks and server impersonation attacks. And then they proposed an improved two-factor authentication protocol for TMIS. In fact, Chaudhry et al's scheme is insecure under lost/stolen smartcard disguised attack and off-line password guessing attack, for that an insider adversary can extract information $\left(r_{i}, h()\right)$ from the memory of the user's smart card. As we generally use passwords which are low-entropy keys, the following attack is feasible in practice: suppose that $P W^{\prime}$ is the guessed password and $l_{i}$ is the user's identity, an insider adversary (e.g. a malicious server) can compute $l_{i}^{\prime}=h\left(I D_{i}\left\|P W^{\prime}\right\| r_{i}\right)$; if $l_{i}^{\prime}=l_{i}$, then the adversary successfully found the correct password $P W_{i}$.

As biometric keys can maintain uniqueness property, they can neither be forged nor guessed easily. Therefore, biometric keys have been widely adpoted in authentication protocols. In 2010, Li and Hwang (2010) proposed a biometric based remote user authentication scheme using user's biometric key to identify the correct user. Li et al. (2011) showed that Li and Hwang's scheme is vulnerable to man-in-the-middle attack, and they proposed an improved biometrics-based remote user authentication scheme. However, Truong et al. (2012) pointed that Li et al's scheme cannot resist stolen verifier attack, reply attack and man-in-the-middle attack, and they proposed an improved remote user authentication scheme. However, the login and password change phase of 
their scheme is not efficient for practice. Later, Awasthi and Srivastava (2013) proposed a new robust biometrics-based remote user authentication scheme using smart cards in order to avoid the time-consuming exponential operations. Unfortunately, Dheerendra et al. (2014) demonstrated that Awasthi et al's scheme fails to resist online and off-line password guessing attack, and they proposed an improved biometrics-based authentication scheme for TMIS. In 2014, He and Wang (2014) proposed a robust multi-server authentication scheme using biometrics-based smart card. But Vanga et al. (2015) pointed that He and Wang's scheme is vulnerable to a known session-specific temporary information attack and impersonation attack. And they proposed a secure biometrics-based multi-server authentication protocol using biometrics-based smart card, and provided simulation results of their scheme for the formal security verification using Automated Validation of Internet Security Protocols and Applications (AVISPA) tool (AVISPA; Lv et al. 2013).

\section{Our contributions}

In this paper, we show that Zhang et al's protocol (Zhang and Zhou 2015) is vulnerable to lost/stolen smartcard disguised attack and off-line password guessing attack. And then we propose an improved protocol using biometric keys (fingerprint, face and palmprint, etc.) to resolve the security problems. Furthermore, we provide the simulation results of our scheme for the formal security verification, using applied pi calculus based formal verification tool ProVerif. Our protocol overcomes the weaknesses of Islam et al's scheme and Zhang et al's scheme, and has the similar efficiency in comparison with their schemes.

The rest of paper is organized as follows: we first review Zhang et al's protocol in second section, and show the security weaknesses of Zhang et al.s protocol in third section. Then, we propose an improved authentication protocol for TMIS is in fourth section. The security analysis of the improved scheme is given in fifth section. We prove the session key secrecy and authentication property using pi calculus based ProVerif in sixth section. In seventh section, we compare security and computation cost between our scheme and other related schemes. We conclude the paper in eighth section.

\section{Review of Zhang et al.'s scheme}

In this section, we review Zhang et al's scheme. There are two participants in Zhang et al.s protocol, patient $U$ and telecare server $S$. Table 1 shows the notations used in this paper.

\section{Initialization phase}

$S$ selects an elliptic curve $E_{p}(a, b)$ over a prime finite field $F_{p}$ and a base point $P$ over $E_{p}(a$, $b)$. Followed that, $S$ chooses a random number $s \in Z_{p}^{*}$ as his secret value, and computes $Q_{s}=s P$, and selects a one-way hash function $H(\cdot):\{0,1\}^{*} \rightarrow Z_{p}^{*}$, and publishes $\left\{E_{p}(a\right.$, b), $\left.P, H(\cdot), Q_{s}\right\}$ and keeps $s$ as a secret value.

\section{Registration phase}

1. $U$ selects his identity $I D$, its password $P W$ and a random number $r$, and computes $l=H(r \| P W)$ and sends $(I D, l)$ to $S$ via a secure way. 
Table 1 The notations

\begin{tabular}{ll}
\hline Notations & Description \\
\hline$U$ & Patient in TMIS \\
$S$ & Telecare server in TMIS \\
$I D$ & Patient U's identity \\
$P W$ & Patient U's password \\
$S$ & Telecare server's secret key \\
$Q_{S}$ & Telecare server's public key, where $Q_{s}=s P$ \\
$E_{k} / D_{k}$ & Symmetric encryption/decryption algorithm with key $k$ \\
$H(\cdot)$ & Secure one-way collision-resistant hash function \\
$\|$ & String concatenation operation \\
$\oplus$ & Exclusive OR operation \\
\hline
\end{tabular}

2. Upon receiving (ID, $l), S$ verifies user's legitimacy in his database. If $I D$ is a new patient, $S$ sets $N=0$, otherwise, $U$ is re-registering to the system, $S$ sets $N=N+1$, and stores $(I D, N, T)$ into its database, where $\mathrm{T}$ is the current registration time.

3. $S$ computes $\sigma=H(s \oplus I D), v=\sigma \oplus l, \mu=H(I D \oplus l)$ and stores $\left\{v, \mu, P, H(\cdot), N, E_{p}(a\right.$, b) \} into the smart card, and sends it to $U$ via a secure way.

4. On obtaining the smartcard, $U$ stores the number $r$ in it.

\section{Login and authentication phase}

1. $U$ inserts his smart card into the terminal and inputs his identity $I D$ and password $P W$. The smartcard computes $l=H(r \| P W), \mu^{\prime}=H(I D \oplus l)$, and checks whether $\mu^{\prime}=\mu$ holds. If not, it aborts the session; otherwise, it selects a random number $a$ and a current timestamp $T_{1}$. Then, smartcard computes $V=a P, I=a Q_{s^{\prime}}$ $K_{u}=H\left(I \| T_{1}\right), \sigma=v \oplus l, D=H(V\|N\| \sigma)$ and $G_{1}=E_{K_{U}}(I D \| D)$. Then, smartcard sends login information $m_{1}=\left\{V, G_{1}, T_{1}\right\}$ to $U$ via the public channel.

2. After receiving $m_{1}$ at $T_{2}, S$ checks whether $T_{2}-T_{1}<\Delta T$ is valid. If it is true, $S$ computes $I=s V, K_{s}=H\left(I \| T_{1}\right)$, and decrypts $G_{1}$ to get $I D^{\prime}$ and $D^{\prime}$, and checks if $I D^{\prime}$ is found in the database. If not, $S$ terminates the session; otherwise, $S$ computes $\sigma^{*}=H\left(s \oplus I D^{\prime}\right)$ and checks whether $D^{\prime}=H\left(V\|N\| \sigma^{*}\right)$ holds. If not, this session terminates; otherwise, $S$ selects a random number $c$ and computes $W=c P, J=c V$, $s k_{s}=H\left(I D^{\prime}\|I\| J\right), G_{2}=H\left(\sigma^{*}\left\|I D^{\prime}\right\| s k_{s}\|W\| T_{2}\right)$, and $S$ sends $m_{2}=\left\{W, G_{2}, T_{2}\right\}$ to $U$ via the public channel. If $T_{2}$ is invalid, abort, otherwise, smartcard computes $J=a W$, $s k_{u}=H(I D\|I\| J), G_{2}^{\prime}=H\left(\sigma\|I D\| s k_{u}\|W\| T_{2}\right)$, and checks whether $G_{2}^{\prime}=G_{2}$ holds. If not, it aborts the session; otherwise, $U$ authenticates $S$ successfully.

\section{Password updating phase}

$U$ inserts his smart card into the terminal and enter his $I D$ and $P W$ when he wants to update its password.

1. The smartcard computes $l=H(r \| P W), \mu^{\prime}=H(I D \oplus l)$, and checks whether $\mu^{\prime}=\mu$ holds. If not, it aborts the session; otherwise, it selects a new random number $r^{*}$ and a new password $P W^{*}$, and updates corresponding value in the smart card. 
2. The smartcard computes $\sigma=v \oplus l, l^{*}=H\left(r^{*} \| P W^{*}\right), v^{*}=\sigma \oplus l^{*}, \mu^{*}=H\left(I D \oplus l^{*}\right)$ and replaces $(\nu, \mu)$ with $\left(v^{*}, \mu^{*}\right)$, respectively.

\section{Lost/stolen smartcard revocation phase}

When $U$ 's smartcard is lost or stolen, it will request $S$ for its revocation.

1. $U$ chooses its new password $P W^{*}$ and new random number $r^{*}$, and computes $l^{*}=H\left(r^{*} \| P W^{*}\right)$, and submits $\left(I D, l^{*}\right)$ to $S$ over a secure channel.

2. $S$ firstly checks the registration credentials of $U$. If the credential provided by $U$ is valid, $S$ updates $N$ as $N=N+1$ for the tuple $\left(I D, N, T_{1}\right)$ to revoke the smartcard.

3. $S$ computes $\sigma=H(s \oplus I D), \quad v^{*}=\sigma \oplus l^{*}, \quad \mu^{*}=H\left(I D \oplus l^{*}\right), \quad$ and stores $\left\{v^{*}, \mu^{*}, P, H(\cdot), Q_{s}, N, E_{p}(a, b)\right\}$ into the smart card, and sends it to $U$ via a secure way.

4. On obtaining the smartcard, $U$ stores the random number $r^{*}$ in it. Finally, the smartcard stores $\left\{r^{*}, v^{*}, \mu^{*}, P, H(\cdot), Q_{s}, N, E_{p}(a, b)\right\}$.

\section{Weaknesses of Zhang et al.'s scheme}

Through careful analysis, we find that Zhang et al's protocol is vulnerable to off-line password guessing attack and lost/stolen smartcard disguised attack. The detailed analyses are described as follows.

\section{Off-line password guessing attack}

If an insider adversary in TMIS can extract information $(r, \mu)$ from the memory of the user's smart card (Zhang and Zhou 2015). Generally speaking, password is not highentropy keys (Abadi and Fournet 2001). Therefore, the following attack is feasible in practice. Suppose that $P W^{\prime}$ is the guessed password, and an insider adversary (e.g. the user's colleague or malicious server) may know the user's identity easily.

The insider adversary in TMIS who knows ID can compute $l^{\prime}=H\left(r \| P W^{\prime}\right)$, $\mu^{\prime}=H\left(I D \oplus l^{\prime}\right)=H\left(I D \oplus H\left(r \| P W^{\prime}\right)\right)$, and checks whether $\mu^{\prime}=\mu$ holds. If it is true, the insider adversary has guessed the correct password. Otherwise, it repeatedly guesses a new password until he succeeds.

\section{Failure to provide the revocation of lost/stolen smartcard}

Though the Zhang et al.s scheme has lost/stolen smartcard revocation phase, an insider adversary can still use the lost/stolen smartcard to pass through the authentication process. The reason is that $\sigma=H(s \oplus I D)$ and $I D$ in the new smart card are the same as that of the lost/stolen smartcard, and $N=N+1$, according to off-line password guessing attack, the adversary can easily get $P W$ and compute the correct authentication request message $m_{1}=\left\{V, G, T_{1}\right\}$, which can pass the authentication of the server.

\section{The improved scheme}

In our improved scheme, $\left\{s, E_{p}(a, b), P, H(\cdot), Q_{s}\right\}$ are the same as that of Zhang et al.s scheme. 


\section{Registration phases}

When a user $U$ wants to become a legal user, he should register to $S$ as follows.

1. $U$ selects his identity $I D$, password $P W$ and a random number $r$, and computes $l=H(r \| P W)$, and sends $(I D, l)$ to $S$ via a secure way.

2. Upon receiving $(I D, l), S$ verifies user's legitimacy in his database. If $I D$ is a new patient, $S$ sets $N=0$, otherwise, $U$ is re-registering to the system, $S$ sets $N=N+1$, and stores the tuple $\left(I D, N, N_{c}\right)$ to its database, where $N_{c}$ is the identity of the smart card.

3. $S$ computes $\alpha=H(s \oplus I D), \beta=\alpha \oplus l$ and stores $\left\{\beta, P, H(\cdot), Q_{s}, N, N_{c}, E_{p}(a, b)\right\}$ into the smart card, and sends it to $U$ via a secure way.

4. On obtaining the smartcard, $U$ scans and enters his personal biometrics Bio. It is worth mentioning that no one can get Bio except $U$ and the biometrics scanner can be combined in the smart card reader. $U$ computes $\mu=r \oplus H(B i o)$, $\theta=H(I D\|P W\| r), U$ stores $(\mu, \theta)$ in the smart card.

\section{Login and authentication phases}

In this phase, the user $U$ and the server $S$ can be authenticated each other and establish the session key $s k$, which showed in Algorithm 1.

1. $U$ inserts his smart card into the terminal and inputs his identity $I D$, password $P W$ and Bio. The smartcard computes $r^{\prime}=\mu \oplus H($ Bio $), \theta^{\prime}=H\left(r^{\prime}\|P W\| I D\right)$, and checks whether $\theta^{\prime}=\theta$ holds. If not, it aborts the session; otherwise, it selects two random numbers $a$ and $N_{1}$. Then, smartcard computes $V=a P, I=a Q_{s}, K_{u}=H\left(I \| N_{1}\right)$, $\alpha=\beta \oplus l, \gamma=H\left(V, N, N_{1}, \alpha, N_{c}\right)$ and $G_{1}=E_{K_{U}}\left(I D\left\|N_{1}\right\| \gamma \| N_{c}\right)$. Then, smartcard sends login information $m_{1}=\left\{V, G_{1}, N_{1}\right\}$ to $S$ via the public channel.

2. After receiving $m_{1}, S$ checks whether $N_{1}$ is a fresh nonce or not. If it is true, $S$ computes $I=s V, K_{s}=H\left(I \| N_{1}\right)$, and decrypts $G_{1}$ to get $I D^{\prime}, N_{c}, \gamma$ and $N_{1}$, and checks whether or not $I D^{\prime}$ is found in the database. If not, $S$ terminates the session; otherwise, $S$ computes $\alpha^{*}=H(s \oplus I D), \gamma^{*}=H\left(V, N, N_{1}, \alpha^{*}, N_{c}\right)$, and checks whether $\gamma^{*}=\gamma$ holds. If is not true, $S$ terminates the session; otherwise, it selects two random numbers $c$ and $N_{2}$ for computing $W=c P, J=c V, K=H\left(J \| N_{2}\right) G_{2}=E_{K}\left(Q_{s} \| N_{2}\right)$, $s k=H\left(I D^{\prime}\left\|Q_{s}\right\| I\|J\| N_{1} \| N_{2}\right)$, and $S$ sends $m_{2}=\left\{W, G_{2}, N_{2}\right\}$ to $U$ via the public channel. If $N_{2}$ is not a fresh nonce number, abort, otherwise, smartcard computes $J=a W, K=H\left(J \| N_{2}\right)$, and decrypts $G_{2}$ to get $Q_{s}$ and $N_{2}$, and checks whether or not $Q_{s}^{\prime}=Q_{s}$ holds. If not, smartcard terminates the session; otherwise, $U$ authenticates $S$ successfully, and computes $s k=H\left(I D\left\|Q_{s}\right\| I\|J\| N_{1} \| N_{2}\right)$. 


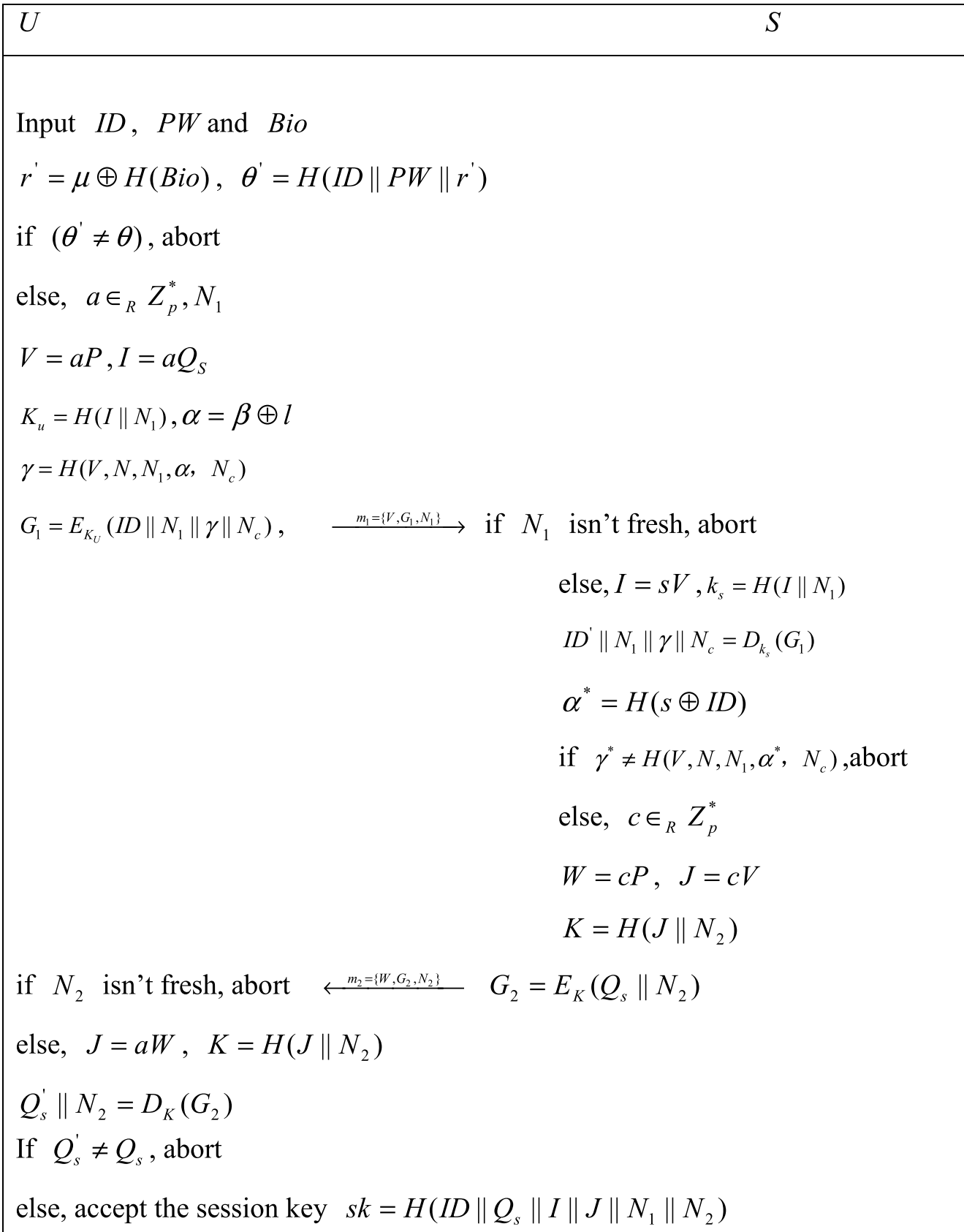

Algorithm 1 Login and authentication phases

\section{Password updating phases}

$U$ inserts his smart card into the terminal and enter his $I D$ and $P W$ when he wants to update its password.

1. The smartcard computes $r^{\prime}=\mu \oplus H(B i o), l=H\left(P W \| r^{\prime}\right), \theta=H\left(I D\|P W\| r^{\prime}\right)$ and checks whether $\theta^{\prime}=\theta$ holds. If not, it aborts the session; otherwise, it selects a new 
random number $r^{*}$ and a new password $P W^{*}$, and updates corresponding value in the smart card.

2. The smartcard computes $\mu^{*}=r^{*} \oplus H(B i o), \theta^{*}=H\left(I D\left\|P W^{*}\right\| r^{*}\right)$ and replaces $(\mu$, $\theta)$ with $\left(\mu^{*}, \theta^{*}\right)$.

\section{Lost/stolen smartcard revocation phases}

When $U$ 's smartcard is lost or stolen, it will request $S$ for its revocation.

1. $U$ chooses its new password $P W^{*}$ and new random number $r^{*}$, and computes $l^{*}=H\left(r^{*} \| P W^{*}\right), \quad \mu^{*}=r^{*} \oplus H(B i o), \quad \theta^{*}=H\left(I D\left\|P W^{*}\right\| r^{*}\right)$ and submits $\left(I D, l^{*}, \mu^{*}, \theta^{*}\right)$ to $S$ over a secure channel.

2. $S$ checks the registration credentials of $U$. If the credential provided by $U$ is valid, $S$ updates $N$ as $N=N+1$ for the tuple $\left(I D, N, N_{c}\right)$ to revoke the smartcard, and deletes $N_{c}$ from his database and selects a new smartcard number $N_{\text {new }}$ for $U$, and returns the tuple $\left(I D, N, N_{\text {new }}\right)$ to his database.

3. $S$ computes $\alpha=H(s \oplus I D), \quad \beta^{*}=\alpha \oplus l^{*}, \quad \theta^{*}=H\left(I D\|P W\| r^{*}\right)$, and stores $\left\{\beta^{*}, P, H(\cdot), Q_{s}, N, N_{\text {new }}, E_{p}(a, b)\right\}$ into the smart card, and sends it to $U$ via a secure way.

4. On obtaining the smartcard, $U$ stores $\left(\mu^{*}, \theta^{*}\right)$ in it. Finally, the smartcard stores $\left\{\theta^{*}, \mu^{*}, \beta^{*}, P, H(\cdot), Q_{s}, N, N_{\text {new }}, E_{p}(a, b)\right\}$.

\section{Security analysis}

In this section, we analyze the security of the improved protocol. The following attacks assume that a malicious adversary can eavesdrop, modify, insert, or delete any messages transmitted via public channel.

The improved protocol can achieve mutual authentication

As $V=a P, I=a Q_{s}, K_{u}=H\left(I \| N_{1}\right)$, and $G_{1}=E_{K_{U}}\left(I D\left\|N_{1}\right\| \gamma \| N_{c}\right)$, only the legal user $U$ can get the secret value $\left(I, N_{1}\right)$ to generate a legal $G_{1} . S$ decrypts $G_{1}$ and checks whether $I D^{\prime}=I D$ holds. If it is true, $S$ can authenticate $U$, otherwise, $U$ cannot be authenticated by $S$. On the other hand, $U$ can authenticate $S$ by verifying whether $Q_{s}^{\prime}=Q_{s}$ hold. As a result, our protocol achieves the mutual authentication.

\section{Malicious insider impersonation attack}

Login phase: If a malicious user $U_{A}$ wants to impersonate $U$, he must forge a valid login message $\left\{V^{*}, G_{1}^{*}, N_{1}\right\} \quad$ where $V^{*}=a^{*} P, \quad I^{*}=a^{*} Q_{s}, \quad K^{*}=H\left(I^{*}|| N_{1}\right), \quad$ and $G_{1}^{*}=E_{K^{*}}\left(I D^{*}\left\|N_{1}\right\| \gamma \| N_{c}\right)$, however, $U_{A}$ can not get $I$, such that it has to forge an invalid one. When $S$ receives the login request message from $U$, it will decrypt and compute $G_{1}^{*}=E_{K^{*}}\left(I D^{*}\left\|N_{1}\right\| \gamma \| N_{c}\right)$, but the equation $I D^{*}=I D$ does not hold, therefore, $S$ will reject the login request. Thus, our scheme can resist insider impersonation attack.

\section{Off-line password guessing attack}

If a malicious attacker has stolen user's smart card, then he can extract the information $\left\{\theta, \mu, \beta, P, H(\cdot), N, Q_{s}, E_{p}(a, b)\right\}$ from the smart card, where $\mu=r \oplus H(B i o)$, $\theta=H(I D\|P W\| r), l=H(r \| P W)$. Since $r$ is protected by Bio and $P W$ is protected by 
a one-way hash function, the attacker cannot know both of the real identity $I D$ and the correct password $P W$. It is impossible to guess these two parameters correctly in polynomial time. Therefore, our protocol is secure against the off-line password guessing attack.

\section{Strong replay attack}

If a malicious attacker wants to replay a previously transmitted message of the sender or the receiver, the attack will fail since $U$ and $S$ choose different random numbers $\left(N_{1}, N_{2}\right)$ in each session. During the authentication phase, after $S$ response the next login message $m_{1}^{\prime}=\left\{V^{\prime}, G_{1}^{\prime}, N_{1}^{\prime}\right\}$ using a valid nonce $N_{1}$, the attacker can neither verify its validness nor obtain the session key assuming the intractability of Diffie-Hellman problem.

\section{Lost/stolen smartcard attack}

When the attacker attempts to insert the lost smart card into the device, it can't pass the authentication of the server, since the stolen card's $N_{c}$ is updated in the database of $S$.

\section{Perfect forward secrecy}

In our protocol, the session key is $s k=H\left(I D\left\|Q_{s}\right\| I\|J\| N_{1} \| N_{2}\right)$, where $I=a Q_{s}=a s P$, $J=c V=c a P$. Since $a$ and $c$ are random numbers chosen by $U$ and $S$, their values are changed in each session run. Therefore, our protocol can provide perfect forward secrecy.

\section{Formal verification}

Some formal verification tools are used to prove the security of cryptographic protocols, such as BAN logic, AVISPA and ProVerif (Abadi et al. 2009). In this section, we prove the session key secrecy and authentication using formal verification tool ProVerif, which is based on applied pi calculus (Abadi and Fournet 2001). The reason is that ProVerif is performed automatically, and the errors can be detected easily, while the formal security proof is artificial structured, and the errors may not easy to be found.

The ProVerif code for the definition of functions, reduction, equation, free names and constants is as follows. 
(* channel$*)$

sch: secure channel between $\mathrm{S}$ and $\mathrm{U}$.

free sch: channel [private].

(*----------------variables and constants-

const ID: bitstring.

const PW: bitstring [private].

const Nc: bitstring [private].

const P: bitstring.

const zero: bitstring.

const one: bitstring.

free s: bitstring [private].

free sk:bitstring [private].

free sku:bitstring [private].

(*----------------constructor-----------------*)

fun $\mathrm{H}$ (bitstring): bitstring.

fun senc(bitstring, bitstring): bitstring.

fun or(bitstring, bitstring): bitstring.

fun $\exp$ (bitstring, bitstring): bitstring.

fun xor(bitstring, bitstring): bitstring.

fun add(bitstring, bitstring): bitstring.

fun mult(bitstring, bitstring): bitstring.

(*---------------destructors \& equations

reduc forall $m$ : bitstring, $n$ : bitstring; $\operatorname{sdec}(\operatorname{senc}(m, n), n)=m$.

equation forall $\mathrm{m}$ : bitstring, $\mathrm{n}$ : bitstring; $\operatorname{xor}(\operatorname{xor}(m, n), n)=m$.

(*----------------------events---------------------------*)

event UserAuthed(bitstring).

event UserStarted(bitstring).

(*---------------------query---------------------------*) 
query attacker(sku).

query attacker(sk).

query id: bitstring; inj-event(UserAuthed(id)) ==> inj-event(UserStarted(id)).

(*-------------------processes--------------------------*)

let $\mathrm{U}=$

new r: bitstring;

let $\mathrm{l}=\mathrm{H}(\mathrm{or}(\mathrm{r}, \mathrm{PW}))$ in

$\operatorname{out}(\operatorname{sch},(\mathrm{ID}, 1))$;

in (sch,(d':bitstring, $\mathrm{P}^{\prime}$ :bitstring, $\mathrm{Qs}$ ': bitstring,N":bitstring,Nc':bitstring));

new Bio: bitstring;

let $\mathrm{u}=\mathrm{xor}(\mathrm{r}, \mathrm{H}(\mathrm{Bio}))$ in

!

(

event UserStarted(ID);

let $\mathrm{r}^{\prime}=\mathrm{xor}(\mathrm{u}, \mathrm{H}(\mathrm{Bio}))$ in

let $\mathrm{u}^{\prime}=\mathrm{H}\left(\operatorname{or}\left(\mathrm{r}^{\prime}, \mathrm{or}(\mathrm{PW}, \mathrm{ID})\right)\right)$ in

if $\mathrm{u}=\mathrm{u}^{\prime}$ then

new a: bitstring;

new N1: bitstring;

let $\mathrm{V}=\operatorname{mult}(\mathrm{a}, \mathrm{P})$ in

let $\mathrm{I}=$ mult( $\left(\mathrm{a}, \mathrm{Qs} \mathrm{s}^{\prime}\right)$ in

let $\mathrm{Ku}=\mathrm{H}($ or $(\mathrm{I}, \mathrm{N} 1))$ in

let $\mathrm{f}^{\prime}=\operatorname{xor}\left(\mathrm{d}^{\prime}, 1\right)$ in

let $\mathrm{ml}=\left(\mathrm{V}, \mathrm{N}^{\prime \prime}, \mathrm{N} 1, \mathrm{f}^{\prime}, \mathrm{Nc}\right)$ in

let $\mathrm{r}^{\prime \prime}=\mathrm{H}(\mathrm{m} 1)$ in

let $\mathrm{G} 1=\operatorname{senc}\left(\operatorname{or}\left(\mathrm{ID}, \operatorname{or}\left(\mathrm{N} 1\right.\right.\right.$, or $\left.\left.\left.\left(\mathrm{r}^{\prime \prime}, \mathrm{Nc}\right)\right)\right), \mathrm{Ku}\right)$ in

out(sch,(V,G1,N1));

in(sch,(W': bitstring,G2": bitstring,N2': bitstring));

let $\mathrm{J}^{\prime}=\operatorname{mult}\left(\mathrm{a}, \mathrm{W}^{\prime}\right)$ in

let $\mathrm{K}^{\prime}=\mathrm{H}\left(\operatorname{or}\left(\mathrm{J}^{\prime}, \mathrm{N} 2^{\prime}\right)\right)$ in 


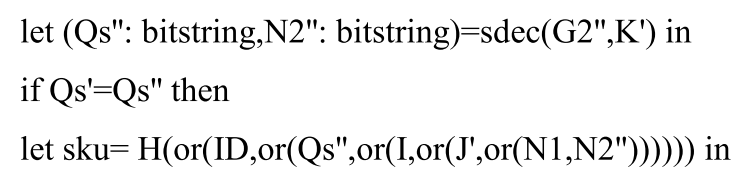

0

) .

let $\mathrm{S}=$

in(sch, (ID': bitstring,l': bitstring));

new N: bitstring;

let $\mathrm{N}^{\prime}=$ if ID' =ID then zero else $\operatorname{add}(\mathrm{N}$, one $)$ in

let $\mathrm{f}=\mathrm{H}\left(\mathrm{xor}\left(\mathrm{s}, \mathrm{ID}^{\prime}\right)\right)$ in

let $\mathrm{d}=\mathrm{xor}\left(\mathrm{f}, \mathrm{l}^{\prime}\right)$ in

let $\mathrm{Qs}=\operatorname{mult}(\mathrm{s}, \mathrm{P})$ in

$\operatorname{out}\left(\mathrm{sch},\left(\mathrm{d}, \mathrm{P}, \mathrm{Qs}, \mathrm{N}^{\prime}, \mathrm{Nc}\right)\right)$;

in(sch,(V': bitstring, G1': bitstring,N1': bitstring));

let $\mathrm{I}^{\prime}=\operatorname{mult}\left(\mathrm{s}, \mathrm{V}^{\prime}\right)$ in

let $\mathrm{Ks}=\mathrm{H}\left(\right.$ or $\left.\left(\mathrm{I}^{\prime}, \mathrm{N} 1^{\prime}\right)\right)$ in

let (ID": bitstring,N1': bitstring,r1: bitstring, Nc: bitstring) $=\operatorname{sdec}\left(\mathrm{G1} 1^{\prime}, \mathrm{Ks}\right)$ in

event UserAuthed(ID");

if ID'=ID" then

let $\mathrm{f} 2=\mathrm{H}\left(\mathrm{xor}\left(\mathrm{s}, \mathrm{ID}^{\prime \prime}\right)\right)$ in

let $\mathrm{m} 2=\left(\mathrm{V}^{\prime}, \mathrm{N}^{\prime}, \mathrm{N} 1{ }^{\prime}, \mathrm{f} 2\right)$ in

let $\mathrm{r} 2=\mathrm{H}(\mathrm{m} 2)$ in

if $\mathrm{r} 1=\mathrm{r} 2$ then

new c: bitstring;

new N2: bitstring;

let $\mathrm{W}=\operatorname{mult}(\mathrm{c}, \mathrm{P})$ in

let $\mathrm{J}=$ mult $\left(\mathrm{c}, \mathrm{V}^{\prime}\right)$ in

let $\mathrm{K}=\mathrm{H}($ or $(\mathrm{J}, \mathrm{N} 2))$ in

let $\mathrm{G} 2=\operatorname{senc}($ or $(\mathrm{Q} s, \mathrm{~N} 2), \mathrm{K})$ in

let sk=H(or(ID",or(Qs, or (I',or(J,or(N1',N2)))))) in

$\operatorname{out}\left(\mathrm{sch},\left(\mathrm{W}, \mathrm{G} 11^{\prime}, \mathrm{N} 2\right)\right)$.

process !U $\mid \mathrm{S}$

We perform the above process in the latest version 1.88 of ProVerif. The performance results as shown in the Fig. 1 . The experimental results show that our scheme is security. 


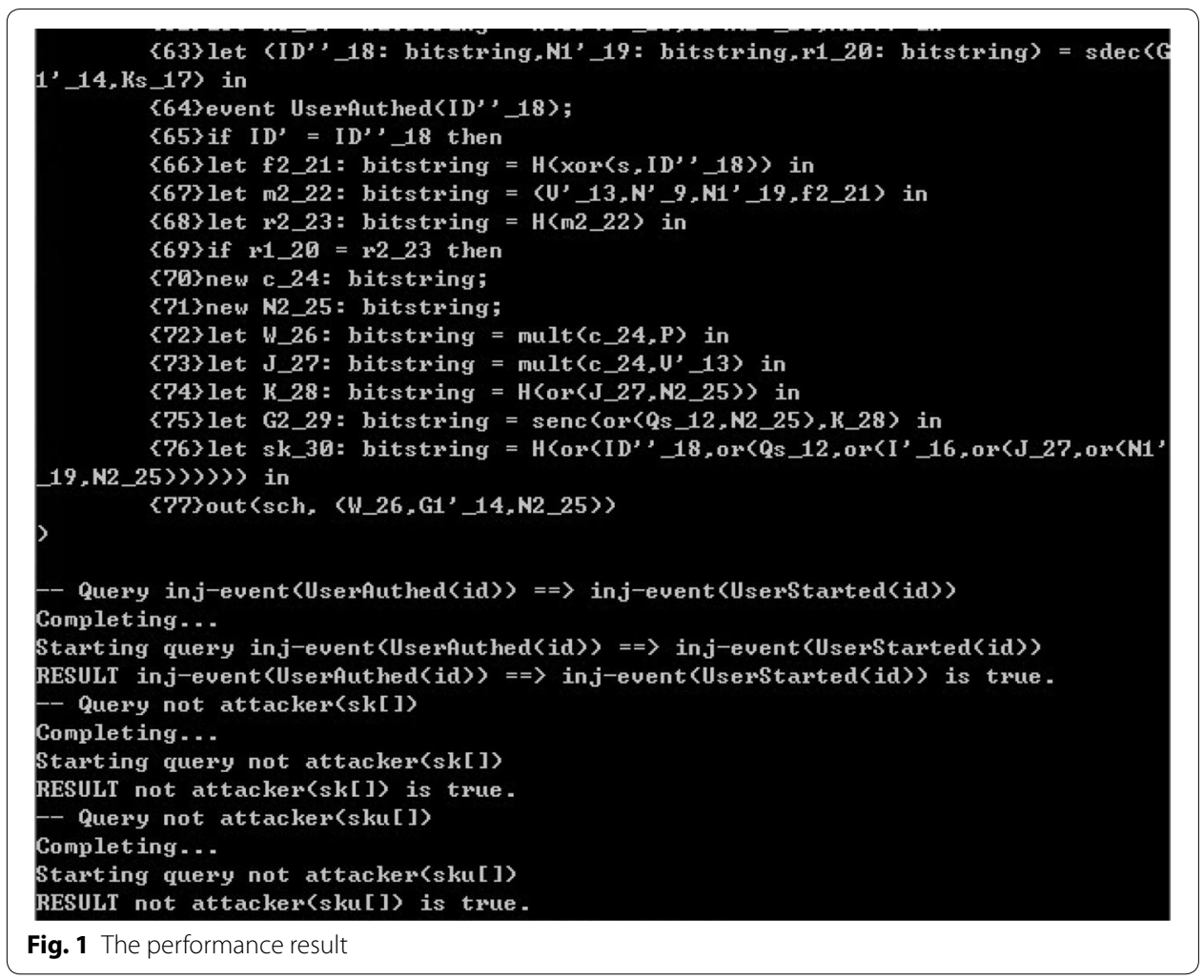

\section{Security and computation cost comparisons}

The security comparison between our scheme and other recently proposed related schemes are given in Table 2.

Let $T_{m}$ be the time complexity of point multiplication in a group, $T_{a}$ be the time complexity of point addition in a group, $T_{s}$ be a symmetric key encryption/decryption operation and $T_{h}$ be a one-way hash operation. Table 3 illustrates the average running times of some commonly used operations estimated by Kilinc and Yanik (2014), and shows that point multiplication in a group is slower than point addition, hash function and symmetric encryption/decryption operation.

Since Islam et al's scheme (Islam and Khan 2014) and Zhang et al's scheme (Zhang and Zhou 2015) are more efficient than other schemes. Therefore, in this section, we only present the computation comparison between our scheme and Islam et al.s and Zhang et al's schemes, and very recently proposed related schemes, which showed in Table 4. From Table 4, we can see that our protocol is almost efficient than that of Zhang et al's and Islam et al.s schemes. However, our protocol overcomes the weaknesses of Islam et al.s and Zhang et al's schemes.

If the scheme can prevent the attack or satisfy the property, the symbol ' $\mathrm{Y}$ ' is used. Otherwise, the symbol ' $N$ ' is used.

\section{Conclusion}

In this paper, we have shown that Zhang et al's protocol cannot achieve some secure properties, including security against off-line password guessing attacks, and it fails to provide the revocation of lost/stolen smartcard. Technically, we adopt random numbers 
Table 2 Security comparison between our scheme and other schemes

\begin{tabular}{|c|c|c|c|c|c|c|c|c|}
\hline $\begin{array}{l}\text { Security } \\
\text { attributes/ } \\
\text { schemes }\end{array}$ & $\begin{array}{l}\text { Li } \\
\text { and Hwang } \\
(2010)\end{array}$ & $\begin{array}{l}\text { Li et al. } \\
\text { (2011) }\end{array}$ & $\begin{array}{l}\text { Truong } \\
\text { et al. } \\
\text { (2012) }\end{array}$ & $\begin{array}{l}\text { Awasthi } \\
\text { and Sriv- } \\
\text { astava } \\
(2013)\end{array}$ & $\begin{array}{l}\text { Dheeren- } \\
\text { dra et al. } \\
\text { (2014) }\end{array}$ & $\begin{array}{l}\mathrm{He} \\
\text { and Wang } \\
(2014)\end{array}$ & $\begin{array}{l}\text { Vanga } \\
\text { et al. } \\
\text { (2015) }\end{array}$ & Ours \\
\hline $\begin{array}{l}\text { Provide } \\
\text { user ano- } \\
\text { nymity }\end{array}$ & $\mathrm{N}$ & $\mathrm{N}$ & Y & Y & $\mathrm{N}$ & $\mathrm{N}$ & Y & $Y$ \\
\hline $\begin{array}{l}\text { Insider } \\
\text { attack }\end{array}$ & $\mathrm{N}$ & Y & Y & Y & $\mathrm{N}$ & Y & Y & $Y$ \\
\hline $\begin{array}{l}\text { Stolen } \\
\text { smart } \\
\text { card } \\
\text { attack }\end{array}$ & $Y$ & Y & Y & Y & $\mathrm{N}$ & Y & Y & $Y$ \\
\hline $\begin{array}{l}\text { Replay } \\
\text { attack }\end{array}$ & $Y$ & Y & Y & Y & Y & $\mathrm{N}$ & Y & $Y$ \\
\hline $\begin{array}{l}\text { Off-line } \\
\text { password } \\
\text { guessing } \\
\text { attack }\end{array}$ & Y & Y & Y & $N$ & Y & $Y$ & Y & $Y$ \\
\hline $\begin{array}{l}\text { Mutual } \\
\text { authenti- } \\
\text { cation }\end{array}$ & $N$ & Y & Y & $\mathrm{N}$ & Y & Y & Y & $Y$ \\
\hline $\begin{array}{l}\text { Known } \\
\text { session- } \\
\text { specific } \\
\text { tempo- } \\
\text { rary infor- } \\
\text { mation } \\
\text { attack }\end{array}$ & $N$ & $N$ & $N$ & $\mathrm{~N}$ & $\mathrm{~N}$ & $\mathrm{~N}$ & Y & $Y$ \\
\hline $\begin{array}{l}\text { Perfect } \\
\text { forward } \\
\text { secrecy }\end{array}$ & $\mathrm{N}$ & $N$ & $N$ & $\mathrm{~N}$ & $\mathrm{~N}$ & Y & Y & $Y$ \\
\hline $\begin{array}{l}\text { Imper- } \\
\text { sonation } \\
\text { attack }\end{array}$ & $\mathrm{N}$ & $N$ & $\mathrm{~N}$ & $\mathrm{~N}$ & $\mathrm{~N}$ & $\mathrm{~N}$ & Y & $Y$ \\
\hline $\begin{array}{l}\text { Provide lost } \\
\text { smart- } \\
\text { card } \\
\text { revoca- } \\
\text { tion }\end{array}$ & N & $\mathrm{N}$ & $\mathrm{N}$ & $\mathrm{N}$ & $\mathrm{N}$ & $\mathrm{N}$ & Y & $Y$ \\
\hline $\begin{array}{l}\text { Server } \\
\text { spoofing } \\
\text { attack }\end{array}$ & $\mathrm{N}$ & $\mathrm{N}$ & $\mathrm{N}$ & $\mathrm{N}$ & $\mathrm{N}$ & Y & Y & $Y$ \\
\hline $\begin{array}{l}\text { Efficient } \\
\text { login } \\
\text { phase }\end{array}$ & $N$ & $N$ & $\mathrm{~N}$ & Y & Y & Y & Y & $Y$ \\
\hline $\begin{array}{l}\text { Efficient } \\
\text { password } \\
\text { change } \\
\text { phase }\end{array}$ & $\mathrm{N}$ & $N$ & $\mathrm{~N}$ & $\mathrm{~N}$ & Y & Y & Y & $Y$ \\
\hline $\begin{array}{l}\text { Biometric } \\
\text { update } \\
\text { phase }\end{array}$ & $\mathrm{N}$ & $\mathrm{N}$ & $N$ & $N$ & $\mathrm{~N}$ & $Y$ & Y & $Y$ \\
\hline
\end{tabular}

Table 3 The running time of different operations

\begin{tabular}{lllll}
\hline Operations & Point multiplication & Point addition & Hash function & $\begin{array}{l}\text { Symmetric } \\
\text { encryption/decryption }\end{array}$ \\
\hline Time $(\mathrm{ms})$ & 2.226 & 0.0288 & 0.0023 & 0.0046 \\
\hline
\end{tabular}


Table 4 Computation cost comparison in login and authentication phase

\begin{tabular}{llllll}
\hline & $\begin{array}{l}\text { Islam and Khan } \\
(\mathbf{2 0 1 4 )}\end{array}$ & $\begin{array}{l}\text { Zhang } \\
\text { and Zhou } \\
(\mathbf{2 0 1 5 )}\end{array}$ & $\begin{array}{l}\text { Chaudhry et al. } \\
\mathbf{( 2 0 1 5 )}\end{array}$ & $\begin{array}{l}\text { Vanga et al. } \\
(\mathbf{2 0 1 5 )}\end{array}$ & Ours \\
\hline $\begin{array}{l}\text { Computational } \\
\text { cost }\end{array}$ & $6 T_{m}+1 T_{a}+10 T_{h}$ & $6 T_{m}+2 T_{s}+11 T_{h}$ & $7 T_{m}+8 T_{h}$ & $5 T_{m}+3 T_{s}+13 T_{h}$ & $6 T_{m}+4 T_{s}+11 T_{h}$ \\
$\begin{array}{c}\text { Estimated time } \\
\text { (ms) }\end{array}$ & 13.4078 & 13.3905 & 15.6004 & 13.4767 & 13.3997 \\
\hline
\end{tabular}

based authentication mechanism, instead of the timestamps that may cause time synchronization problem. An improved protocol is proposed in order to overcome those weaknesses. The simulation results show that when compared with existing protocols, our protocol provides the same level of efficiency and better security guarantees for TMIS applications.

\section{Authors' contributions}

Conceived and designed the experiments: QX. Performed the experiments: $\mathrm{BH}$. Analyzed the data: SBW. Contributed reagents/materials/analysis tools: WHL, QX. Wrote the paper:WHL, QX, SBW. Designed the scheme and wrote the paper:WHL, QX, SBW. Proved the authentication and security of the proposed scheme: $\mathrm{BH}$. Verified the authentication and security of the proposed scheme in the latest version 1.88 of ProVerif: $\mathrm{BH}$. All authors read and approved the final manuscript.

\section{Authors' information}

Wenhao Liu received the Ph.D. degree from University of Electronic Science and Technology of China in 2010. Currently, he is a lecturer in the school of Information Science and Engineering, Hangzhou Normal University, China. His research area is applied cryptography, including digital signatures, cloud computing security and key agreement protocols etc. Qi Xie is a professor in Key Laboratory of Cryptography and Network Security, Hangzhou Normal University, China. He received his Ph.D. degree in applied mathematics from Zhejiang University, China, in 2005. He was a visiting scholar between 2009 and 2010 at Department of Computer Science, University of Birmingham in UK, and a visiting scholar to the Department of Computer Science at City University of Hong Kong in 2012. His research area is applied cryptography, including digital signatures, authentication and key agreement protocols etc. He has published over 60 research papers in international journals and conferences, and served as co-chair of ISPEC 2012 and ASIACCS 2013. Shengbao Wang received his PhD degree in computer science from Shanghai Jiao Tong University in 2008 and is now working as an associate professor at the Department of Computer Science and Engineering, Hangzhou Normal University, China. His research interests lie in the area of public key cryptography, especially focus on public key encryption and key agreement protocols. Bin Hu received the Ph.D. degree from Zhejiang University in 2009, China. Currently, he is a lecturer in the school of Information Science and Engineering, Hangzhou Normal University, China. His research mainly concerns reasoning about communication protocols, especially verification of their properties. In particular, he works on logic and calculus based specification and verification of protocols.

\section{Acknowledgements}

This research was supported by Natural Science Foundation of Zhejiang Province (No. LZ12F02005), the Major State Basic Research Development (973) Program of China (No. 2013CB834205), and the National Natural Science Foundation of China (No. 61103209).

\section{Competing interests}

The authors declare that they have no competing interests.

Received: 11 August 2015 Accepted: 16 March 2016

Published online: 03 May 2016

\section{References}

Abadi M, Fournet C (2001) Mobile values, new names, and secure communication. In: Proceedings of the 28th ACM SIGPLAN-SIGACT symposium on principles of programming languages. ACM New York, 2001, pp. 104-115

Abadi M, Blanchet B, and Lundh HC (2009) Models and proofs of protocol security: a progress report. In: 21st international conference on computer aided verification, Grenoble, France, 2009, pp 35-49

Automated Validation of Internet Security Protocols and Applications (AVISPA). http://www.avispa-project.org/. Accessed 6 Jan 2015

Awasthi AK, Srivastava K (2013) A biometric authentication scheme for telecare medicine information systems with nonce. J Med Syst 37(5):1-4 
Chaudhry S, Naqvi H, Shon T (2015) Cryptanalysis and improved two factor authentication protocol for telecare medicine information systems. J Med Syst 39:66

Chen H, Luo J, Yeh C (2012) An efficient and secure dynamic id-based authentication scheme for telecare medical information system. J Med Syst 36(6):3907-3915

Dheerendra M, Sourav M, Saru K (2014) Security enhancement of a biometric based authentication scheme for telecare medicine information systems with nonce. J Med Syst 38:41

He DB, Wang D (2014) Robust biometrics-based authentication scheme for multi-server environment. IEEE Syst J. doi:10.1109/JSYST.2014.2301517

He DB, Chen JH, Zhang R (2012) A more secure authentication scheme for telecare medicine information systems. J Med Syst 36(3):1989-1995

Islam SH, Biswas GP (2014) Cryptanalysis and improvement of a password-based user authentication scheme for integrated EPR information system. J King Saud Univ Comput Inf Sci 25:51-61

Islam S, Khan M (2014) Cryptanalysis and improved of authentication and key agreement protocols for telecare medicine information systems. J Med Syst 38:135

Jiang Q, Ma J, Ma Z (2013) A privacy enhanced authentication and key agreement scheme for telecare medicine information systems. J Med Syst 37:8979

Kaul SD, Awasthi AK (2013) RFID authentication protocol to enhance patient medication safety. J Med Syst 37:9979

Khan SU, Lavagno L, Pastrone C (2014) Online authentication and key establishment scheme for heterogeneous sensor networks. Int J Distrib Sens Netw 2014. Article ID 718286

Kilinc HH, Yanik T (2014) A survey of SIP authentication and key agreement schemes. IEEE Commun Surv Tutor 16(2):1005-1023

Li CT, Hwang MS (2010) An efficient biometrics-based remote user authentication scheme using smart cards. J Netw Comput Appl 33(1):1-5

Li X, Niu JW, Ma J (2011) Cryptanalysis and improvement of a biometrics-based remote user authentication scheme using smart cards. J Netw Comput Appl 34(1):73-79

Lv C, Ma M, Li H (2013) An novel three-party authenticated key exchange protocol using one-time key. J Netw Comput 36(1):498-505

Muhaya FT (2015) Cryptanalysis and security enhancement of Zhu's authentication scheme for telecare medical information systems. Secur Commun Netw 8(2):149-158

Pu Q, Wang J, Zhao R (2010) Strong authentication scheme for telecare medicine information systems. J Med Syst 36(4):2609-2619

Siddiqui Z, Abdullah AH, Khan MK, Alghamdi AS (2014) Smart environment as a service: three factor cloud based user authentication for telecare medical information system. J Med Syst 38:9997

Truong TT, Tran MT, Duong AD (2012) Robust biometrics based remote user authentication scheme using smart cards. In: 15th IEEE international conference on network-based information systems (NBiS'2012), pp 384-391

Vanga O, Ashok K, Adrijit G (2015) A secure biometrics-based multi-server environment authentication protocol using smart cards. IEEE Trans Inf Forensics Secur 10(9):1953-1966

Wei J, Hu X, Liu W (2012) An improved authentication scheme for telecare medicine information systems. J Med Syst 36(6):3597-3604

Wen F (2013) A robust uniqueness-and-anonymity-preserving remote user authentication scheme for connected health care. J MedSyst 37:9980

Wu ZY, Lee YC, Lai F, Lee HC, Chung Y (2010) A secure authentication scheme for telecare medicine information systems. J Med Syst 36(3):1529-1535

Wu ZY, Chung Y, Lai F, Chen TS (2012) A password-based user authentication scheme for the integrated EPR information system. J Med Syst 36(2):631-638

Xie Q, Zhang J, Dong N (2013) Robust anonymous authentication scheme for telecare medical information systems. J Med Syst 37(2):1-8

Xie Q, Liu WH, Wang SB (2014) Improvement of a uniqueness-and-anonymity-preserving user authentication scheme for connected health care. J Med Syst 38(9):91

Xu X, Zhu P, Wen Q (2014) A secure and efficient authentication scheme for telecare medicine information systems. J Med Syst 38:9994

Zhang L, Zhou S (2015) Roubust ECC-based authenticated key agreement scheme with privacy protection for telecare medicine information systems. J Med Syst 39:49

Zhu ZA (2012) An efficient authentication scheme for telecare medical information platform. J Med Syst 36(6):3833-3838 